\title{
Dynamic model for the simulation of surge in a centrifugal compression system with vaned diffuser
}

\author{
Yang Zhao \\ Xi'an Jiaotong University \\ zhaoyangzyang@stu.xjtu.edu.cn \\ Xi'an, Shaanxi, China \\ Pengfei Zhang \\ Xi'an Jiaotong University \\ zhangpengfeicn89@stu.xjtu.edu.cn \\ Xi'an, Shaanxi, China
}

\author{
Zhiheng Wang \\ Xi'an Jiaotong University \\ wangzhiheng@mail.xjtu.edu.cn \\ Xi'an, Shaanxi, China \\ Guang Xi \\ Xi'an Jiaotong University \\ xiguang@xjtu.edu.cn \\ Xi'an, Shaanxi, China
}

\begin{abstract}
As the flow rate decreases from stable point to surge point, the flow phenomenon of low-frequency system dependent pulsation occurs in a centrifugal compressor compression system. In order to predict the system instability of surge with higher accuracy, the mathematical model of pipeline coupled with the model of lumped parameters is used to investigate the effect of pipeline on the system instability of a centrifugal compressor. The predicted vibration frequency of mild surge matches well with the measured data and the relative error is just $2.3 \%$. And also, the spatial distribution of gas fluctuation in pipeline is analysed in detail to elucidate the developing characteristics of system instability affected by the piping system. Moreover, with the analysis of system instability under varied parameters of pipeline, each parameter has a different influence on the stability and vibration frequency. This study confirms that this model is a useful tool to give a detailed analysis about the effect of pipeline on system instability.
\end{abstract}

\section{Introduction}

The centrifugal compressors are widely used as the core components of industrial products such as aero-engines, gas turbine and turbocharger. When the operating point is gradually modified from the chock point to lower mass flow rate, the stable operation of the compression system is generally restricted by the occurrence of system instability such as surge. And it can be characterized as axisymmetric disturbance oscillating in the whole system. The periodical fluctuation of mass flow rate and pressure ratio can have a significant influence on the many aspects of the compression system such as affecting the performance of downstream components and inducing the destructive failure of the blade or shaft bearing. More and more researchers have paid much effort to investigate the characteristics of surge.

Due to the destructiveness of surge, it is required to investigate the flow instability of surge to minimize its effect or to propose proper control method. So many published papers are about developing the simplified model to predict the system instability. Based on the theoretical model of lumped parameters firstly proposed by Greitzer(1976) for a axial compressor, it is generally believed that dynamic instability of surge occurs when the slope of the compressor characteristic is higher than the critical value $1 /\left(B^{2} T^{\prime}\right)$. Then the model was validated to be suitable for the centrifugal compressor by Hansen(1981). And also, compared with the experimental data, Fink et al.(1992) took the fluctuation of rotating speed into consideration to develop the model, accurately predicting the observed behaviour. But the model is originally applied for the compressor system consisting of three parts such as an actuator, inlet/outlet duct and a big plenum. The major assumption of the model is onedimensional incompressible flow in the duct and isentropic compression in the plenum. Such assumption is just suitable for the compressor with a large plenum. However, the compressor is generally coupled with complex piping system instead of a large plenum. Moreover, the interaction of the compressor and piping system can have a determined influence on the system instability. Tamaki et al.(2008) took experimental measurement to evaluate the effect of piping system on the operating range. But they still used the model of lumped parameter to predict system instability. When the surge occurs in the compressor stage, the flow in the component such as the upstream and downstream duct generally presents one-dimensional characteristic (Bozza, 2011). In order to accurately predict the unsteady behaviour of the compression especially the acoustic waves in pipelines, Yoon et al.(2011) took the dynamics of the piping system into consideration to enhance the Greitzer model. Galindo (2011) used the method of characteristics to solve the one-dimensional flow in piping system. And Denher (2016) used the one-dimensional CFD to predict the unsteady behaviour of surge with piping system. Moreover, Zhang (2019) used a novel 1D-3D coupled method to predict the surge boundary of a centrifugal compressor, considering both 
the transient flow characteristics in duct and compressor. But few published papers have taken a comprehensive investigation on the effect of piping system on the system instability.

As mentioned before, more and more researches tend to conduct the simulation in high order to predict the system instability accurately. However, compared with onedimensional numerical simulation of piping system, the model based on the theory of planar wave has the advantage of simplicity and effectiveness to determine the system dynamic. So the mathematical model of pipeline coupled with the model of lumped parameters is used to investigate the time-domain and spatial feature of the unsteady behaviour. This paper is organized into four parts. First, a brief introduction about the test stage is given. Second, it is about the compressor model in detail. The third part is the result discussion. And then several conclusions are given finally.

\section{Experimental rig}

The test stage is a large-scale and low-speed centrifugal compression system established by National Engineering Research Centre of Fluid Machinery \& Compressors of Xi'an Jiaotong University. The centrifugal compressor consists of inlet guide vane, an unshrouded impeller, an airfoil vaned diffuser and a volute. The main geometric data of the test stage is shown in Table 1. And also, Table 2 shows the design parameters of the compressor. At the operating condition, as the scheme of compressor test rig shows in Figure 1, the filtered air flows through the inlet duct, the centrifugal compressor, outlet duct, exit throttle valve and then is blown into the atmosphere. The measurement of the compressor performance has been conducted over the operating range from chock point to mild surge point. More detail about the measurement of the stage measurement can be found in the previous paper (Zhao et al., 2017). The blade angle is taken from the tangential direction. The operated condition can be modified by the exit throttle. The length and area of the inlet/outlet duct has been shown in Table 3.

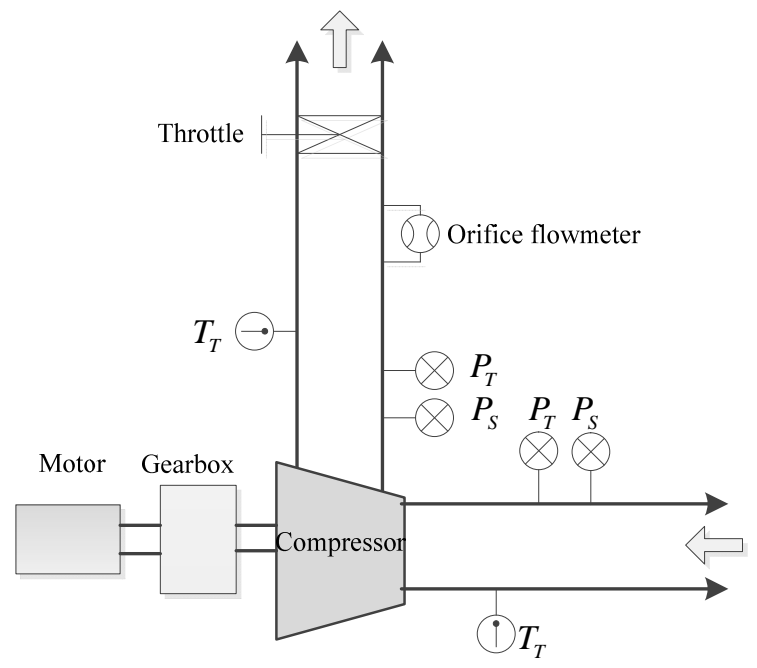

Figure 1 Scheme of compressor test rig
Table 1 Geometric data of the test rig

\begin{tabular}{cccc}
\hline \hline Parameters & IGV & Impeller & $\begin{array}{c}\text { Vaned } \\
\text { diffuser }\end{array}$ \\
\hline Inlet diameter (mm) & 423 & 421.8 & 940 \\
Outlet diameter & 423 & 796 & 1258 \\
(mm) & 115 & 103.9 & 43.8 \\
Inlet span (mm) & 115 & 43.8 & 43.8 \\
Outlet span (mm) & 0 & 28 & 17 \\
Inlet angle(deg) & 0 & 58 & 29 \\
Outlet angle (deg) & 13 & $10+10$ & 13 \\
Blade number &
\end{tabular}

Table 2 Design specification of the test rig

\begin{tabular}{cc}
\hline \hline Parameters & Value \\
\hline Blade tip Mach number & 0.515 \\
Design speed $(\mathrm{r} / \mathrm{min})$ & 4320 \\
Tip clearance $(\mathrm{mm})$ & 1.5 \\
Design mass flow rate $(\mathrm{kg} / \mathrm{s})$ & 5.8 \\
Total pressure ratio & 1.26 \\
\hline \hline
\end{tabular}

Table 3 Geometrical data of the compression system

\begin{tabular}{ccc}
\hline \hline Component & $\mathrm{L}(\mathrm{m})$ & $\mathrm{A}\left(\mathrm{m}^{2}\right)$ \\
\hline Inlet duct & 5.5 & 0.139 \\
Exit duct & 8.6 & 0.113 \\
\hline \hline
\end{tabular}

In order to measure the unsteady behaviour of surge, a high frequency response pressure transducer, Kulite LQ-47, was embedded in the diffuser blade at about $5 \%$ chord and mid-span of the pressure surface. Then NI data acquisition model PXI-643 was used to acquire the dynamic data. The sample frequency was set to $140 \mathrm{KHz}$ in order to be able to capture both the blade passing and surge frequency.

\section{Compressor model}

In order to predict the dynamic behaviour of centrifugal compression system, the model of lumped parameters based on the conservation of mass and momentum is generally used as the following equations show.

$$
\begin{gathered}
\left(\frac{L}{A}\right)_{c} \frac{d m_{c}}{d t}=\Delta P_{c}-\Delta P_{P} \\
\frac{V_{P}}{c^{2}} \frac{d \Delta P_{P}}{d t}=m_{c}-m_{t}
\end{gathered}
$$

Where the fluid in the duct is regarded to be impressible and the fluid in the plenum is under isentropic compression. But for the compressor with long pipelines especially at compressor outlet, the acoustic waves and flow pulsation in the pipelines has a determined influence on the dynamic behaviour of the system. So it is incorrect to use such model by simply transforming the piping system into duct-plenum system. So the following models are given to predict the dynamics of each component in the compression system with pipelines.

\section{A. Transfer matrix of inlet and outlet duct}

When the magnitude of pressure pulsation is less than $8 \%$ of mean value of pressure, the transfer matrix method based on the theory of planar wave (Krus et al., 1994) can be used to describe the gas fluctuation and acoustic waves. 
Based on the continuity, momentum and wave equations, the transient disturbance of pressure and velocity in a duct can be given as the following equations:

$$
\begin{aligned}
& p_{t}^{*}=A^{*} e^{j w\left(t-\frac{x}{c+u_{0}}\right)}+B^{*} e^{j w\left(t+\frac{x}{c-u_{0}}\right)} \\
& u_{t}^{*}=\frac{1}{c \rho_{0}}\left(A^{*} e^{j w\left(t-\frac{x}{c}\right)}-B^{*} e^{j w\left(t+\frac{x}{c}\right)}\right)
\end{aligned}
$$

Where $\mathrm{A}$ and $\mathrm{B}$ are complex constants. And $w$ is the complex frequency.

Then the transfer matrix of the disturbance between the inlet and any position in the duct is given by the equation (5).

$$
\left[\begin{array}{l}
p_{x}^{*} \\
u_{x}^{*}
\end{array}\right]=\left[\begin{array}{cc}
\cos \frac{w}{c} x & -j \rho_{0} c \sin \frac{w}{c} x \\
-\frac{j}{\rho_{0} c} \sin \frac{w}{c} x & \cos \frac{w}{c} x
\end{array}\right]\left[\begin{array}{l}
p_{1}^{*} \\
u_{1}^{*}
\end{array}\right]
$$

Moreover, the transfer matrix in time-domain can be transformed into that in the frequency domain to make it easier to be solved, as the equation (6) shows.

$$
\left[\begin{array}{c}
p_{2} \\
-u_{2}
\end{array}\right]=\left[\begin{array}{cc}
\cosh \left(\frac{s}{c_{0}} x\right) & -\rho_{0} c_{0} \sinh \left(\frac{s}{c_{0}} x\right) \\
-\frac{1}{\rho_{0} c_{0}} \sinh \left(\frac{s}{c_{0}} x\right) & \cosh \left(\frac{s}{c_{0}} x\right)
\end{array}\right]\left[\begin{array}{l}
p_{1} \\
u_{1}
\end{array}\right]
$$

\section{B. Transfer matrix of component with different} diameters

For the component with different diameters meaning different area, the transfer matrix can be given as following equation.

$$
\left[\begin{array}{l}
p_{2}^{*} \\
u_{2}^{*}
\end{array}\right]=\left[\begin{array}{ll}
1 & 0 \\
0 & \frac{s_{1}}{s_{2}}
\end{array}\right]\left[\begin{array}{l}
p_{1}^{*} \\
u_{1}^{*}
\end{array}\right]
$$

\section{Transfer matrix of time-lagging effect of the compressor}

For the compressor, the time-lagging effect between transient and steady-state performance can be given as the equation (8). Then the transfer matrix is given as the equation (9).

$$
\begin{gathered}
\tau \cdot \frac{d\left(\overline{p_{2}}-\overline{p_{1}}\right)}{d t}=\varphi\left(\overline{u_{1}}\right)-\left(\overline{p_{2}}-\overline{p_{1}}\right) \\
{\left[\begin{array}{l}
p_{2}^{*} \\
u_{2}^{*}
\end{array}\right]=\left[\begin{array}{cc}
1 & \frac{\varphi^{\prime}}{1+j w \tau} \\
0 & 1
\end{array}\right]\left[\begin{array}{l}
p_{1}^{*} \\
u_{1}^{*}
\end{array}\right]}
\end{gathered}
$$

Where $\tau$ means the throughflow time of compressor.

D. Transfer matrix of inertial effect of the compressor

Based on the inertial equation (10) in the flow passage of the compressor, the transfer matrix of flow disturbance can be given by equation (11).

$$
\begin{gathered}
p_{t 1}-p_{t 2}=\rho_{0} s_{1}\left(\frac{L}{A}\right)_{c} \frac{\partial u_{t 1}}{\partial t} \\
{\left[\begin{array}{l}
p_{2}^{*} \\
u_{2}^{*}
\end{array}\right]=\left[\begin{array}{cc}
1 & -j w \rho_{0} s_{1}\left(\frac{L}{A}\right)_{c} \\
0 & 1
\end{array}\right]\left[\begin{array}{l}
p_{1}^{*} \\
u_{1}^{*}
\end{array}\right]}
\end{gathered}
$$

Where $(L / A)$ is given with the summation of that in each component including the IGV, impeller and vaned diffuser.

\section{E. Transfer matrix of throttle}

Based on the throttle equation $p_{1}-p_{2}=\lambda u_{1}^{2}$, the transfer matrix of disturbance is given by the equation (12).

$$
\left[\begin{array}{l}
p_{2}^{*} \\
u_{2}^{*}
\end{array}\right]=\left[\begin{array}{cc}
1 & -2 \lambda u_{0} \\
0 & \frac{s_{1}}{s_{2}}
\end{array}\right]\left[\begin{array}{l}
p_{1}^{*} \\
u_{1}^{*}
\end{array}\right]
$$

Based on the transfer matrix given in the compressor model, the relation between the disturbance at the system inlet and outlet boundary can be given as the equation.

$$
\left[\begin{array}{c}
p_{\text {out }}^{*} \\
u_{\text {out }}^{*}
\end{array}\right]=M_{T} M_{D 2} \cdots M_{D 1}\left[\begin{array}{c}
p_{\text {in }}^{*} \\
u_{\text {in }}^{*}
\end{array}\right]
$$

Combined with the inlet boundary condition $p_{1}^{*}=0$, the pressure fluctuation at system outlet can be given as $p_{\text {out }}^{*}=u_{\text {in }}^{*} f(w)$. According to the outlet boundary condition, we can get the equation (14).

$$
p_{\text {out }}^{*}=u_{\text {in }}^{*} f(w)=0
$$

Then the complex frequency can be solved as $w=w_{i}+j w_{j}$. Breaking the equation (14) into its real and imaginary parts, the equations become $\operatorname{Re}(f(w))=0$ and $\operatorname{Im}(f(w))=0$. The solution can be graphically determined by the intersection of zero-contours of the hypersurfaces. The real part of the solution means the vibration frequency of disturbance and the imaginary part shows the attenuation rate of the disturbance, indicating the stability of the system. If $w_{j}>0$, the disturbance magnitude will decrease with an exponential rate like $e^{-w_{j} t}$ and the system is unstable. If not, the disturbance magnitude will increase with an exponential rate and then the system is unstable.

\section{Results and discussion}

\section{Validation of the surge model}

In the experimental measurement, low-frequency pressure fluctuation of mild surge occurs when the mass flow is gradually decreased to be $3.0 \mathrm{~kg} / \mathrm{s}$ as the Figure 2 shows. And the frequency analysis of the unsteady pressure in Figure 3 shows that there exists three peaks of frequency responding to the BPF $(1467 \mathrm{~Hz})$, half harmonic of BPF $(733 \mathrm{~Hz})$ and the surge frequency $(3.8 \mathrm{~Hz})$. 


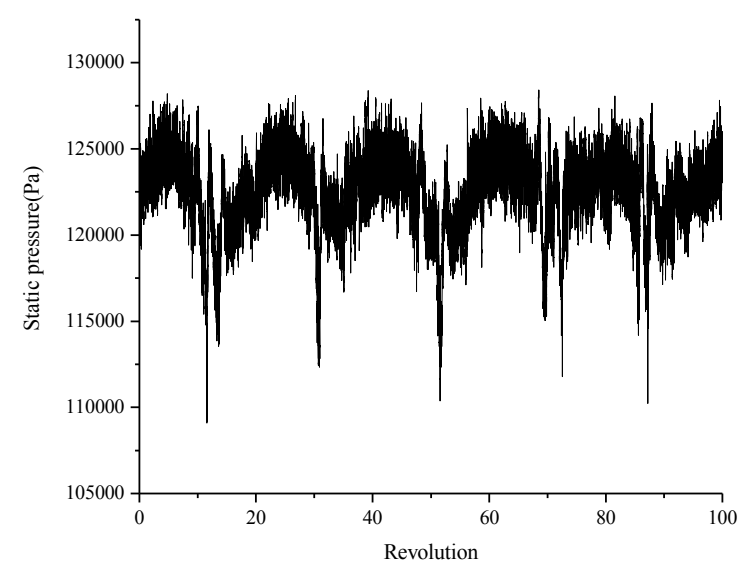

Figure 2 Unsteady static pressure at the 5\% chord of the pressure surface of the diffuser blade

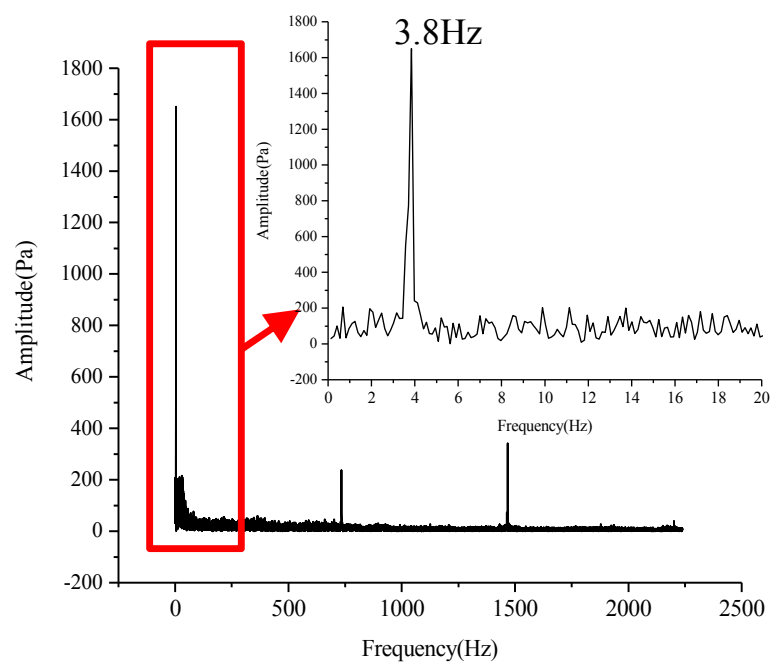

Figure 3 FFT analysis of the unsteady pressure

As mentioned in the compressor model, with the fixed geometry of the test stage, the system stability depends on the slope of the characteristic and throttle parameter. At the operating point $3.0 \mathrm{~kg} / \mathrm{s}$, if the slope of the characteristic is gradually modified with a fixed step (as shown in Figure 4), the system turns to be unstable gradually. And also, the vibration frequency decreases at the same time. In the figure, the point A responses to the limit unstable point in experimental measurement. It shows the vibration frequency of point A to be $3.89 \mathrm{~Hz}$. The prediction of the frequency of mild surge is within $2.3 \%$ of measured value, showing a good agreement with the experimental data.

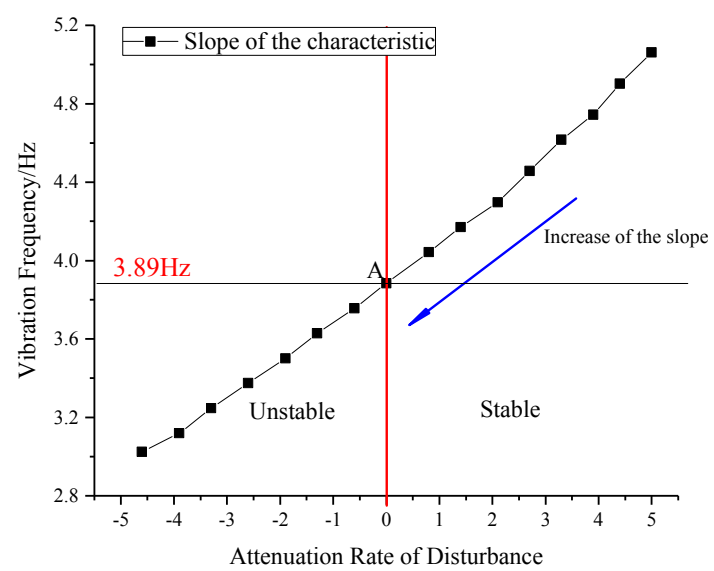

Figure 4 Prediction of system stability and vibration frequency

\section{Spatial distribution of fluctuation of pressure and velocity}

As mentioned before, the acoustic waves and flow pulsation in the pipelines have been taken into consideration to accurately predict the system stability of the compression system. So there exists spatial inhomogeneous of pressure and velocity disturbance in the piping system.

For the system inlet, the fluctuation magnitude of pressure and velocity can be set to be zero and per unit respectively. Based on the transfer matrix, the magnitude and phase of the disturbance can be given by the Figure 5 and 6 .

As the distribution of phase angle shows in the Figure 6, the phase angle of pressure and velocity disturbance keeps constant and the pressure disturbance is lagged with the velocity in 90 degree. There exists large magnitude of spatial inhomogeneity as shown in Figure 5. The magnitude of the pressure disturbance increases gradually to reach the maximum $u_{1} \rho_{i n} c_{i n} \sin \frac{w}{C} L_{i n}$ at the end of inlet-duct, which can also be solved by the transfer matrix of inlet duct. The inhomogeneity of velocity disturbance is much smaller, reaching the minimum $u_{1} \cos \frac{w}{C} L_{i n}$ at the end of the inlet duct. When the vibration frequency is small or the inlet duct is short, $\frac{w}{c} L_{i n}$ will be relatively small that the velocity disturbance at inlet duct is approximately uniform. Combined with the uniform mean flow velocity in the inlet duct, the internal flow velocity can be considered to be uniform, meaning $\partial u_{k} / \partial x_{k}=0$. Based on the continuity equation $D \rho / D t+\rho \partial u_{k} / \partial x_{k}=0$ and $\partial u_{k} / \partial x_{k}=0$, the uniform flow can be considered to be incompressible for $D \rho / D t=0$. Therefore the inlet duct can be solved with the momentum equation $(L / A)_{i n} d m / d t=p_{1}-p_{2}$.

For the compressor, because of the combined effect of time-lagged performance, inertial balance and acoustics in volute, the phase angle of pressure is increased obviously and 
the phase angle of velocity is reduced in a low magnitude. Moreover, the magnitude of pressure is increased clearly but the magnitude of the velocity is almost not changed. The obvious variation of pressure disturbance is mainly caused by the positive slope $\varphi^{\prime}$ of the pressure-rise characteristic as shown in equation (9).

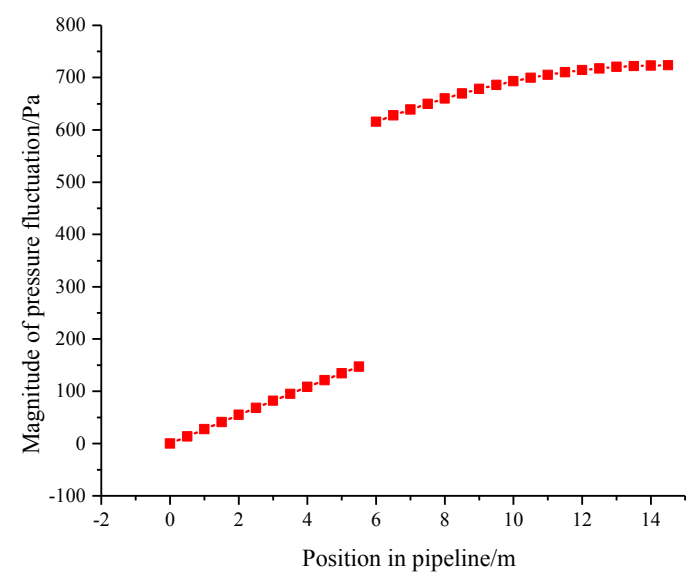

a. Pressure

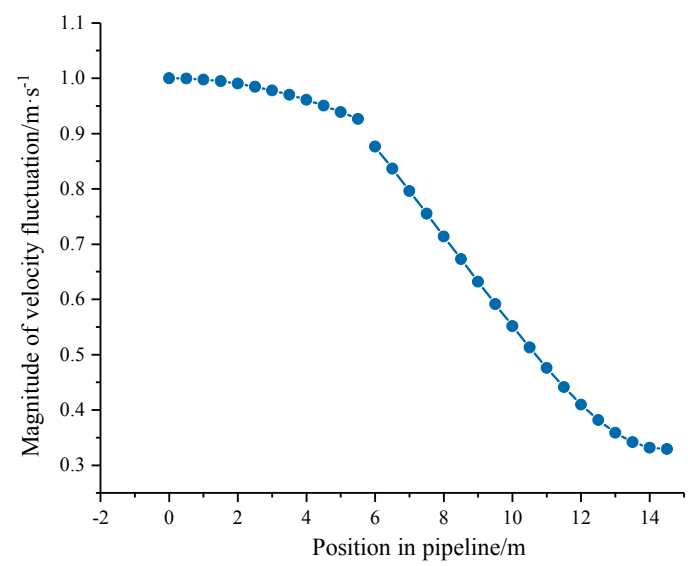

b. Velocity

Figure 5 Distribution of magnitude of disturbance in the piping system

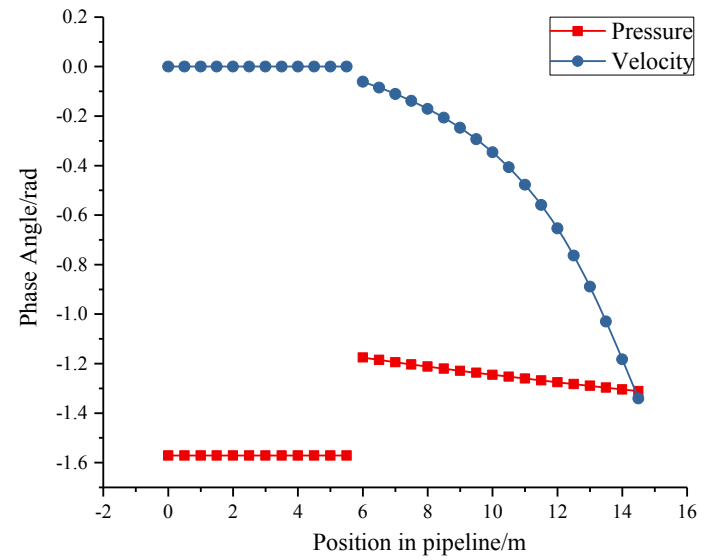

Figure 6 Distribution of phase angle of disturbance in the piping system
For the outlet pipeline, the spatial variation of magnitude of pressure disturbance is relatively smaller than that of inlet pipeline while the distribution of magnitude of velocity presents the obviously different variation.

In general condition, for the outlet pipeline with the assumption of incompressible fluid, the equation (15) shows that the velocity disturbance lags behind the pressure by 90 degree.

$$
\left(\frac{L}{A}\right)_{\text {out }} \frac{d m}{d t}=p_{1}-p_{2}
$$

For a plenum with the assumption of isentropic compression, the equation (2) shows that the velocity disturbance leads the pressure by 90 degree. But for the outlet duct considering the acoustic effect, the Figure 6 shows that although the velocity disturbance lags behind the pressure disturbance, the phase-lagged angle is changing obviously. It may be caused by the higher effect of plenum than the inertial effect in outlet duct.

\section{Effect of variation of geometry on the system instability}

As mentioned in the introduction, the geometry of the piping system has a determined influence on the system stability. So the effect of geometry's variation on the stability and vibration frequency has been investigated in this part.

Based on the original test rig, four parameters of the inlet and outlet pipelines are increased or decreased with a step of a quarter of original value. And the Figure 7 shows the system instability and vibration frequency under different geometry of piping system.

a. When the length of the inlet duct is increased or the area is reduced, the system stability is improved and vibration frequency is reduced. As mentioned before, the inertial effect reflecting by the parameter $(\mathrm{L} / \mathrm{A})$ plays a much more determined influence on the gas fluctuation in the inlet duct. When the parameter is increased, the inertial effect becomes larger that it increases the system stability and decreases the frequency as $B=\frac{U}{2 c} \sqrt{\frac{V_{p}}{(L / A)_{s} A_{c}^{2}}}$ and $f=\frac{c}{2 \pi} \sqrt{\left(\frac{A}{L}\right)_{s} \frac{1}{V_{p}}}$ show (Greitzer, 1976).

b. When the length and area of the outlet duct is increased, the vibration frequency will be reduced and the system will become unstable from stable condition. It is because the plenum effect is much stronger than the inertial effect. The increase of length can make both the plenum effect and inertial effect stronger, but the former is much stronger than latter. So it will induce the stability to be weaker and the frequency to be smaller.

At the same time, the increase of the area at outlet duct will decrease the inertial effect and increase the plenum effect. Then the compressor system will encounter stronger instability. And the vibration frequency is also decreasing but with a smaller magnitude compared with the decreasing length of outlet duct. 


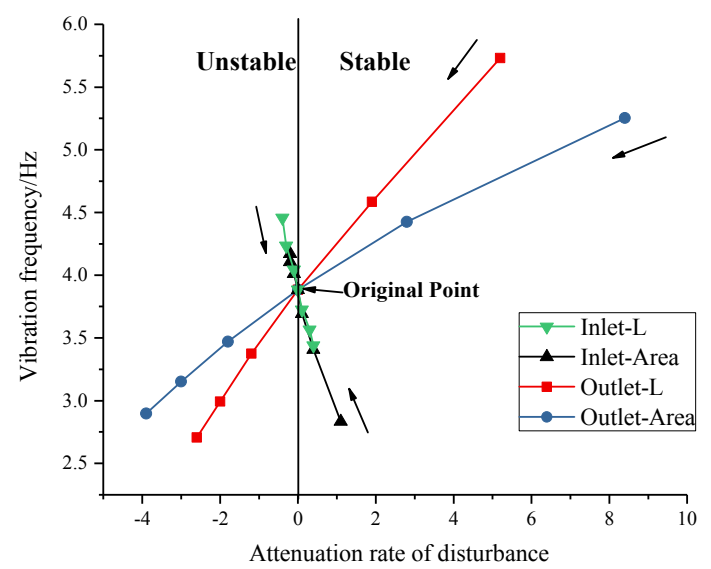

Figure 7 Effect of different geometry of pipeline on system instability

\section{Transient fluctuation of mass flow rate and velocity in} mild surge

Based on the mathematical model of the compression system mentioned before, the model of each component can be built in the Simulink code of Matlab software to simulate the dynamic behaviour of mild surge. At the surge point $3.0 \mathrm{~kg} / \mathrm{s}$, it is initialized from the equilibrium point with a small pulse disturbance at 0.5 second. As shown in the Figure 8, the disturbance will increase gradually to develop into mild surge. The magnitude of developed velocity and pressure fluctuation is relatively small, just about $2 \mathrm{~m} / \mathrm{s}$, $1500 \mathrm{~Pa}$ respectively at the middle position of inlet and outlet duct.

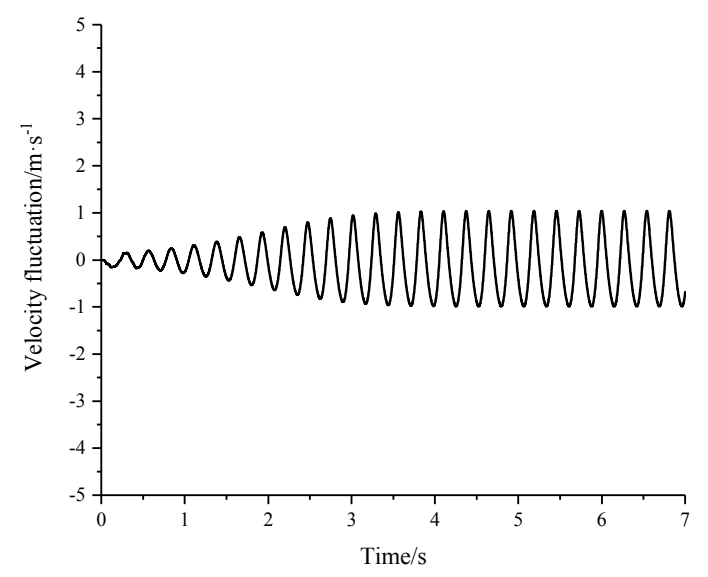

a. Velocity fluctuation at middle of inlet duct

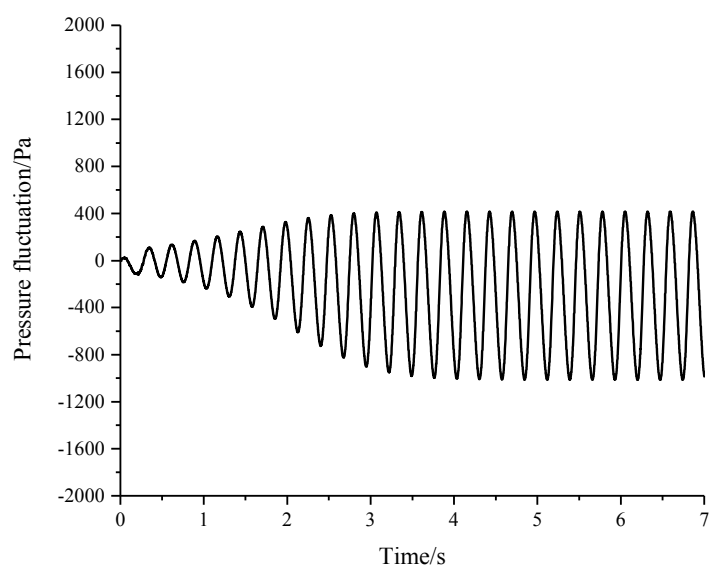

b. Pressure fluctuation at middle of outlet duct Figure 8 Prediction of pressure and velocity fluctuation

\section{CONCLUSIONS}

In the paper, the mathematical model of pipeline coupled with the model of lumped parameters is used to investigate the effect of pipeline on the system instability of a centrifugal compressor. After the detailed analysis of the system instability, several conclusions can be given below.

(1) The mathematical model considering the gas fluctuation and acoustics can accurately predict the vibration frequency at the limit stable point. The relative error between the prediction and measured data is just $2.3 \%$.

(2) There exists obviously spatial inhomogeneity of pressure and velocity disturbance in the piping system.

(3) For the inlet duct, the system instability mainly depend on the parameter (L/A). For the outlet duct, the parameters $\mathrm{L}$ and $\mathrm{A}$ affect the system instability with the similar trend. But the parameter $\mathrm{L}$ affects vibration frequency much more obviously than A but affects stability weakly than A.

(4) The dynamic behavior of gas fluctuation modeled by the method of transfer matrix can be successfully simulated.

\section{Acknowledgement}

The work is financially supported by National Key Research and Development Program of China (Grant No. 2018YFB0606102) and National Nature Science Foundation of China (Grant No. 51576153), which are highly acknowledged.

\begin{tabular}{ll}
\multicolumn{2}{l}{ Nomenclature } \\
$\mathrm{A}$ & Area \\
$\mathrm{B}$ & Dimensional parameter \\
$\mathrm{L}$ & Length \\
$\mathrm{L} / \mathrm{A}$ & Equivalent length-to-area ratio \\
$\mathrm{P}$ & Pressure \\
$\mathrm{s}$ & Sectional area of component \\
$\mathrm{t}$ & Time \\
$\mathrm{u}$ & Velocity
\end{tabular}




$\begin{array}{ll}\text { V } & \text { Volume } \\ \text { BPF } & \text { Blade passing frequency } \\ C & \text { Sound velocity } \\ f & \text { Frequency } \\ \varphi & \text { Steady-state performance } \\ \rho & \text { Density } \\ \lambda & \text { Throttle parameter } \\ w & \text { Complex frequency } \\ \tau & \text { Throughflow time in compressor } \\ X & \text { Axial distance } \\ \Delta P & \text { Pressure rise }\end{array}$

\section{Subscripts}

$\begin{array}{ll}\mathrm{c} & \text { Compressor } \\ \mathrm{d} & \text { Duct } \\ \mathrm{p} & \text { Plenum } \\ \mathrm{t} & \text { Throttle } \\ \text { in } & \text { System inlet } \\ \text { out } & \text { System outlet } \\ 0 & \text { Ambient reference } \\ 1 & \text { Inlet at each component } \\ 2 & \text { Outlet at each component }\end{array}$

\section{References}

1. Greitzer E. M. (1976). Surge and rotating stall in axial flow compressors-Part I: Theoretical Compression System Model. Journal of Engineering for Gas Turbines \& Power, 98, 190.

2. Hansen K. E., Larsen P. S. (1981). Experimental and theoretical study of surge in a small centrifugal compressor. Journal of Fluids Engineering, 103(3), 391395.

3. Fink D. A., Cumpsty N. A., Greitzer E. M. (1992). Surge dynamics in a free-spool centrifugal compressor system. Journal of Turbomachinery, 114(2), 321-332.

4. Tamaki H. (2008). Effect of piping systems on surge in centrifugal compressors. Journal of Mechanical Science and Technology, 22, 1857-1863.

5. Bozza F, De Bellis V, Marelli S, et al. (2011). 1D simulation and experimental analysis of a turbocharger compressor for automotive engines under unsteady flow conditions. SAE International Journal of Engines, 4(1): 1365-1384.

6. Yoon S. Y., Lin Z., Goyne C. et al. (2011). An enhanced Greitzer compressor model with pipeline dynamics included. Journal of Vibration \& Acoustics, 133, 051005.

7. Galindo J., Arnau F. J., Tiseira A., et al. (2011). Solution of the turbocompressor boundary condition for onedimensional gas-dynamic codes, Mathematical \& Computer Modelling an International Journal, 52(7): 1288-1297.

8. Dehner R., Selamet A., Keller P. et al. (2016). Simulation of deep surge in a turbocharger compression system. Journal of Turbomachinery, 138, 111002.

9. Zhang, M., Zheng, X., Huang, Q., \& Sun, Z. (2018). A Novel 1D-3D Coupled Method to Predict Surge
Boundary of Centrifugal Compressors. Journal of Engineering for Gas Turbines and Power, 141(7): 071012.

10. Zhao J. Y., Wang Z. H., Zhao Y. et al.(2018). Investigation of transient flow characteristics inside a centrifugal compressor for design and off-design conditions. Proceedings of the Institution of Mechanical Engineers Part A Journal of Power \& Energy, 232 (4), 364-385.

11. Krus P., Weddfelt K. et al.(1994). Fast pipeline models for simulation of hydraulic systems. ASME Journal of Dynamic Systems Measurements and Control, 116,132136.

12. Greitzer E. M. (1976). Surge and rotating stall in axial flow compressors-part i: Theoretical compression system model, Journal of Engineering for Gas Turbines \& Power, 98 (2): 190. 